\title{
Is Xpert MTB/RIF appropriate for diagnosing tuberculous pleurisy with pleural fluid samples? A systematic review
}

\author{
Zhen-yu Huo and Li Peng*
}

\begin{abstract}
Background: Tuberculous pleurisy (TP) presents a diagnostic problem due to the limitations of traditional diagnostic methods. Different studies with the Xpert MTB/RIF assay have drawn variable conclusions about its values in TP diagnosis. We conducted a meta-analysis to assess whether the Xpert MTB/RIF assay is appropriate for the diagnosis of TP using pleural fluid samples.

Methods: A systematic search of four literature databases in English and Chinese language was performed to identify studies involving the use of Xpert MTB/RIF in patients with TP confirmed by plural biopsy and/or mycobacterial culture. Pooled sensitivity, specificity and accordance proportion were calculated, and the forest plots were generated to assess the accuracy of Xpert MTB/RIF for TP diagnosis.

Results: We identified 23 studies meeting our inclusion criteria. The pooled sensitivity and specificity of Xpert MTB/ RIF were $30 \%\left(95 \% \mathrm{Cl}: 21-42 \%, \mathrm{I}^{2}=87.93 \%\right)$ and $99 \%\left(95 \% \mathrm{Cl}: 97-100 \%, \mathrm{I}^{2}=96.20 \%\right)$, respectively, and the area under the SROC curve (AUC) of Xpert MTB/RIF was 0.86 (95\% Cl: 0.83-0.89). Compared with drug susceptibility testing (DST), the pooled accordance rate of Xpert MTB/RIF in detecting rifampicin-susceptible cases and rifampicinresistant cases was 99\% (95\% Cl: $\left.95-104 \%, \mathrm{I}^{2}=8.7 \%\right)$ and 94\% (95\% Cl: 86-102\%), respectively.

Conclusions: Our analysis suggests that the Xpert MTB/RIF assay is of limited value as a screening test for TP but has a high potential for confirming TP diagnosis and differentiating TP from non-TB diseases using pleural fluid samples.
\end{abstract}

Keywords: Xpert MTB/RIF, Tuberculous pleurisy, Pleural fluid, Rifampicin resistance, Systematic review

\section{Background}

Tuberculosis (TB) remains a serious, life-threatening disease worldwide, with nearly 10.4 million cases and 1.7 million deaths reported in 2016 by the World Health Organization (WHO) [1]. While pulmonary TB is the most common presentation, extra-pulmonary TB is also an important clinical problem. One of the most common types of extra-pulmonary TB is tuberculous pleurisy (TP), which accounts for about one fourth of all TB cases [2]. At present, diagnosis of TP depends largely on detection of Mycobacterium tuberculosis in pleural fluid or pleura by microbiological culture, or demonstration

\footnotetext{
* Correspondence: pli1228@163.com

Department of Respiratory and Critical Care Medicine, the First Affiliated Hospital of Chongqing Medical University, No.1, Youyi Road, Yuzhong District, Chongqing Municipality 400016, China
}

of caseous granulomas in pleura by histopathological examination. However, these methods are invasive, laborious, time-consuming and insensitive, which often delay diagnosis and treatment.

Over the past several years, there has been a significant increase in using the Xpert MTB/RIF assay (also referred to as Xpert; Cepheid Inc., USA), which is an automated, cartridge-based nucleic acid amplification test for TB. This assay has the ability to simultaneously detect $M$. tuberculosis nucleic acid and resistance to rifampin (RIF) in less than $2 \mathrm{~h}$. Due to its excellent performance, this assay has been recommended by WHO for diagnosing $\mathrm{TB}$ and detecting rifampicin resistance in pulmonary and extra-pulmonary $\mathrm{TB}$ in adults and children as well as for initial screening of individuals suspected of having multiple drug resistant-TB (MDR-TB)

(c) The Author(s). 2018 Open Access This article is distributed under the terms of the Creative Commons Attribution 4.0 International License (http://creativecommons.org/licenses/by/4.0/), which permits unrestricted use, distribution, and 
and HIV-co-infected TB cases [3, 4]. Most of the reported studies on Xpert MTB/RIF have been performed in sputum samples from pulmonary TB while there are relatively scarce reports using other types of samples from extra-pulmonary TB.

A limited number of studies have reported the utility of Xpert MTB/RIF in diagnosing TP, with highly variable sensitivity and specificity, ranging from 13 to $100 \%$, between studies [5-7]. In one study carried out in pleural tissue samples from 17 patients with TP, Xpert MTB/RIF failed to detect any TP cases [8]. It is known that mycobacterial culture has limited ability to detect TP, when the study used only a culture reference standard and without histological biopsy, it is likely to overestimate the sensitivity of Xpert and underestimate the specificity $[5,9]$. Although there is a published meta-analysis on the performance of Xpert MTB/RIF in diagnosing TP [10], this study has the limitations of using non-stringent inclusion criteria for TP patients (particularly without histopathological findings) and not including data on rifampicin-resistance. Therefore, the applicability of Xpert MTB/RIF to the diagnosis of TP as well as the detection of rifampicin-resistance in TP patients remains largely unclear.

To better understand the value of Xpert MTB/RIF in TP diagnosis, we conducted a comprehensive meta-analysis of literature published up to May 2018 involving the use of Xpert MTB/RIF for detecting TB and rifampicin resistance in TP patients.

\section{Methods}

Our meta-analysis was presented with reference to the recommendations from the PRISMA statement [11]. All data involved in this analysis were extracted from published articles, therefore, ethical approval was not applicable in this study.

\section{Data sources and search strategy}

A systematic search about studies of the accuracy of the Xpert MTB/RIF in diagnosing TP and rifampicin resistance was carried out. We searched the EMBASE, Cochrane, MEDLINE (PubMed) and China Science and Technology Journal (CSTJ) databases to identify original research articles and conference abstracts in English or Chinese language published on or before May 25, 2018. Search was implemented by using combinations of the following items: "pleural tuberculosis", "tuberculous pleuritis", "tuberculous pleural effusion", "TPE", "Xpert MTB/RIF", "GeneXpert", "Xpert", and "TB/RIF".

\section{Reference standard and study selection}

Our literature search was restricted to studies involving the use of Xpert MTB/RIF in patients diagnosed as TP according to the current gold standard: a combination of histopathological examination and mycobacterial culture $[2,12]$. That is: the reference standard of confirmed TP should include positive Mycobacterium tuberculosis culture from pleural fluid or tissue, or/ and histological manifestations of granulomas in pleural tissue.

As the first step, two investigators (ZY.Huo and L.Peng) independently screened for articles containing the defined items in the title and abstract. All articles reporting the performance of Xpert MTB/RIF in diagnosing TP and rifampicin resistance on pleural fluid samples were retrieved for full-text review. Through full-text review, articles were excluded if they met any of the following criteria: 1) Experiments were not performed with a commercial Xpert MTB/RIF assay; 2) The performance of Xpert MTB/RIF was not evaluated; 3) TP cases were not defined using a combination of histopathological examination and mycobacterial culture; 4) Xpert MTB/RIF was performed using blood or non-pleural fluid specimens; 5) Duplicated reports from the same research group; 6) Reports of systematic reviews or meta-analysis. After full-text review, the two researchers met together to compare the retrieved articles meeting the inclusion criteria. In case of any discrepancy, a third person (physician from our hospital) was invited to discuss and resolve the discrepancy.

\section{Data extraction and quality assessment}

Two researchers (ZY.Huo and L.Peng) independently extracted and crosschecked the data from all included articles. The following information was retrieved from all included articles: 1) The first author, publication year and study location; 2) The number and age of the enrolled participants; 3) The proportion of HIV-seropositive participants; 4) The specimen types and diagnostic standard; 5) The statistics of positive and negative results of the Xpert MTB/RIF assay; 6) The statistics of rifampicin sensitive and resistant cases for the Xpert MTB/RIF assay. The quality of all included articles was evaluated based upon the recommendations from QUADAS-2 checklist [13]. Based on the QUADAS-2 system, concerns with respect to applicability and risk of bias in meta-analysis were verbalized as "high", "unclear" or "high". Two researchers (ZY.Huo and L.Peng) independently scored and recorded all included references using the QUADAS-2 tool, and then reviewed the results together. Next, IBM SPSS software 19.0 (SPSS Inc., USA) was used to calculate the kappa statistic for consistency check. In case of discrepancy, a third person (statistician from our university) was invited to re-evaluate the data and solve the disagreement.

\section{Statistical analysis}

We performed descriptive statistics and analyses adopting the recommended methods for assessment of 
diagnostic trials in meta-analysis [14, 15]. All data analysis including the pooled sensitivity, specificity, and accordance proportion with corresponding 95\% confidence intervals $(95 \% \mathrm{CI})$, and the $\mathrm{I}^{2}$ statistic test was performed using the Stata/MP 13.1 (Stata Corp., USA). The results were summarized and synthesized by using forest plots. A symmetric receiver operator characteristic (SROC) curve was made to present the individual assessment of sensitivity and specificity for each study [16-18]. The publication bias of inclusive researches was evaluated by Deeks' funnel plot asymmetry test.

\section{Results}

Literature search results and characteristics of published studies

Initial literature search resulted in a total of 486 unique studies. Following full-text review, we identified 23 studies that met all search criteria and were suitable for meta-analysis $[5-9,19-36]$. Details of the literature selection process are illustrated in Fig. 1. The characteristics of the 23 included studies are summarized in Table 1. All these 23 studies demonstrated the performance of Xpert MTB/RIF in detecting TB or rifampicin resistance in TP patients confirmed following the gold standard involving a combination of mycobacterial culture and histopathological examination of pleural samples. Publication years ranged from 2011 to 2018 (Table 1).
Study locations included 11 countries across Europe, South America, Asia and Africa. There was a total of 2646 individuals with pleural effusion, including 1194 (45.1\%) with confirmed TP and 1452 (54.9\%) without TP. Of the 23 studies, 7 reported the utility of Xpert MTB/ RIF in HIV-associated TB patients, and 2 reported results of Xpert MTB/RIF in detection of rifampicin resistance in comparison with drug susceptibility testing (DST) $[9,31]$ (Table 2).

\section{Quality assessment of the included studies}

Based on quality assessment by the QUADAS-2 tool, 11 of the 23 included studies showed a high risk of bias, whereas 5 of them had high applicability concerns. The kappa statistic between the primary results of two researchers (ZY.Huo and L.Peng) was $0.787 \quad(\mathrm{P}<0.01)$, which suggested a good inter-rater reliability of consistency. Additional information about the ratings of risk of bias and applicability concerns was provided in Additional file 1.

\section{Sensitivity and specificity of Xpert MTB/RIF}

A total of 2646 eligible participants were used to evaluate the performance of Xpert MTB/RIF for TP diagnosis, including 1194 participants confirmed to be TP by mycobacterial culture and/or histopathological examination, and 1452 participants not diagnosed as TP by the

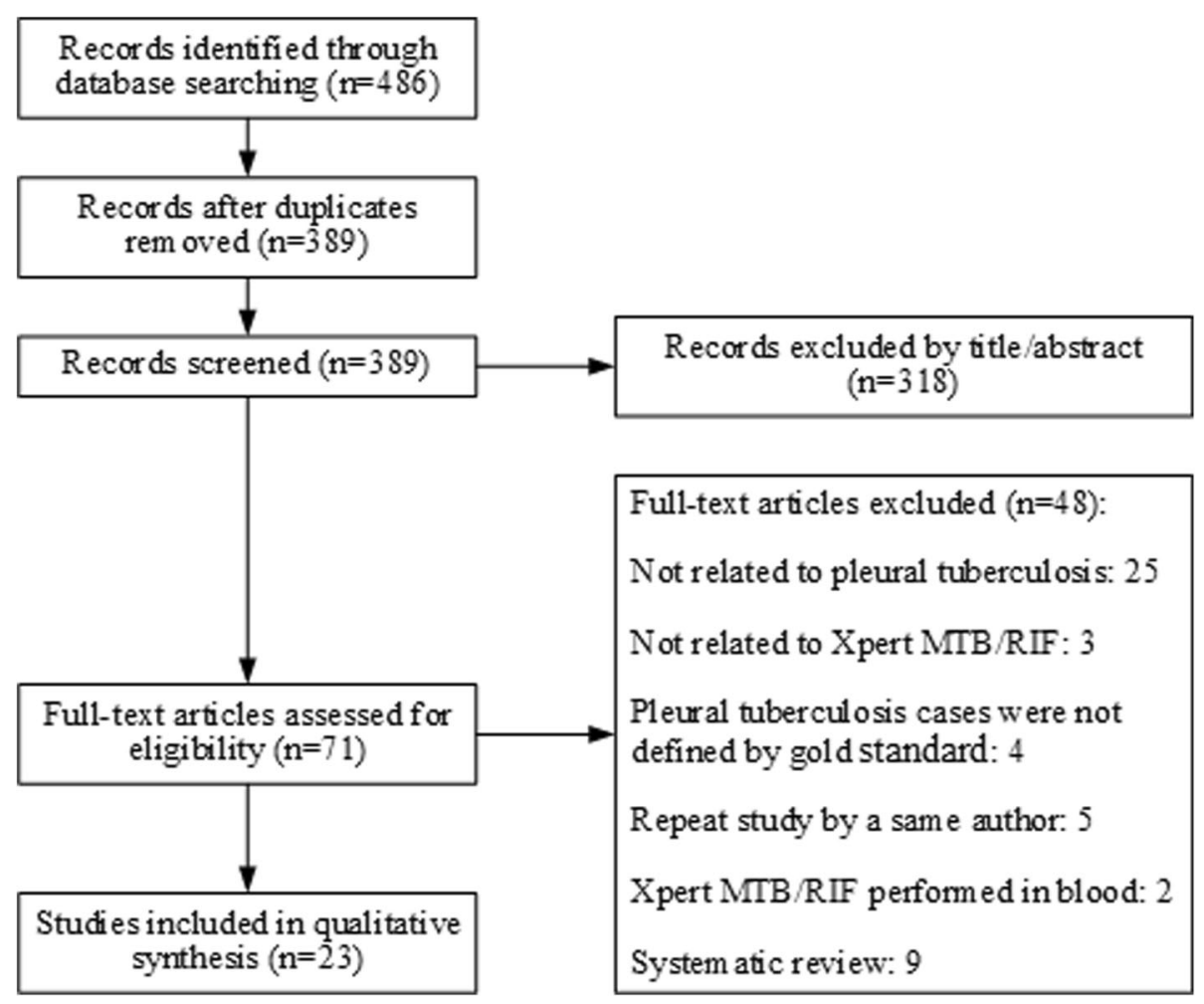

Fig. 1 Flowchart diagram of the literature search process 
Table 1 Characteristics of 23 published studies included for meta-analysis and the primary results of Xpert MTB/RIF test

\begin{tabular}{|c|c|c|c|c|c|c|c|c|c|c|c|}
\hline \multirow[t]{2}{*}{ First author } & \multirow[t]{2}{*}{ Year } & \multirow[t]{2}{*}{ Country } & \multirow{2}{*}{$\begin{array}{l}\text { Patients } \\
\text { enrolled }\end{array}$} & \multirow{2}{*}{$\begin{array}{l}\text { Mean age } \\
\text { (years) }^{a}\end{array}$} & \multirow{2}{*}{$\begin{array}{l}\text { HIV-infection } \\
\text { prevalence (\%) }\end{array}$} & \multirow{2}{*}{$\begin{array}{l}\text { Specimen } \\
\text { types }\end{array}$} & \multirow{2}{*}{$\begin{array}{l}\text { Reference } \\
\text { standard }\end{array}$} & \multicolumn{4}{|c|}{ Results } \\
\hline & & & & & & & & $\overline{\mathrm{TP}}$ & $\mathrm{FP}$ & $\mathrm{FN}$ & TN \\
\hline Causse & 2011 & Spain & 34 & $45(5-83)$ & NR & Pleural fluid & Culture & 4 & 0 & 0 & 30 \\
\hline Friedrich & 2011 & South Africa & 25 & NR & NR & Pleural fluid & Culture & 5 & 0 & 15 & 5 \\
\hline Malbruny & 2011 & France & 12 & 52 & $4.3 \%$ & Pleural fluid & Culture & 0 & 0 & 2 & 10 \\
\hline Moure & 2012 & Spain & 31 & NR & NR & Pleural fluid & Culture & 7 & 0 & 19 & 5 \\
\hline Tortoli & 2012 & Italy & 330 & NR & NR & Pleural fluid & Culture & 5 & 3 & 10 & 312 \\
\hline Christopher & 2013 & India & 91 & $46(33-57)$ & NR & Pleural fluid & Culture and biopsy & 4 & 0 & 26 & 61 \\
\hline Porcel & 2013 & Spain & 67 & 50 & $0 \%$ & Pleural fluid & Culture and biopsy & 5 & 0 & 28 & 34 \\
\hline Zmak & 2013 & Croatia & 42 & NR & NR & Pleural fluid & Culture & 0 & 0 & 1 & 41 \\
\hline Javed & 2014 & Pakistan & 25 & NR & NR & Pleural fluid & Biopsy & 2 & 0 & 12 & 11 \\
\hline Lusiba & 2014 & Uganda & 116 & $34 \pm 13$ & $44.8 \%$ & Pleural fluid & Culture and biopsy & 25 & 1 & 62 & 28 \\
\hline Meldau & 2014 & South Africa & 88 & 51 & $10.2 \%$ & Pleural fluid & Culture and biopsy & 9 & 1 & 31 & 47 \\
\hline Scott & 2014 & South Africa & 528 & 39 & NR & Pleural fluid & Culture & 227 & 3 & 255 & 43 \\
\hline Sharma SK & 2014 & India & 364 & NR & NR & Pleural fluid & Culture & 37 & 8 & 54 & 265 \\
\hline Theron & 2014 & South Africa & 76 & $55(38-65)$ & $17 \%$ & Pleural fluid & Culture & 5 & 6 & 11 & 54 \\
\hline Trajman & 2014 & Brazil & 59 & 50 & $5 \%$ & Pleural fluid & Culture and biopsy & 1 & 0 & 32 & 26 \\
\hline Coleman & 2015 & Malawi & 50 & 32 & $100 \%$ & Pleural fluid & Culture & 9 & 0 & 4 & 18 \\
\hline Liu & 2015 & China & 126 & $38.6 \pm 13.2$ & $4.0 \%$ & Pleural fluid & Culture & 24 & 1 & 31 & 70 \\
\hline Rufai & 2015 & India & 162 & $41.6 \pm 19$ & $0 \%$ & Pleural fluid & Culture & 23 & 0 & 19 & 119 \\
\hline Wang & 2015 & China & 125 & 43 & NR & Pleural fluid & Culture & 13 & 47 & 0 & 65 \\
\hline Che & 2017 & China & 78 & $44(18-83)$ & $1.3 \%$ & Pleural fluid & Culture and biopsy & 12 & 0 & 48 & 18 \\
\hline Li & 2018 & China & 70 & $42 \pm 20$ & $0 \%$ & Pleural fluid & Culture and biopsy & 6 & 0 & 39 & 25 \\
\hline Sharma S & 2018 & India & 37 & 39 & $0 \%$ & Pleural fluid & Culture and biopsy & 5 & 0 & 2 & 30 \\
\hline Christopher & 2018 & India & 130 & $50.9 \pm 14.1$ & $0 \%$ & Pleural fluid & Biopsy & 9 & 0 & 56 & 65 \\
\hline
\end{tabular}

TP, true positive. FP, false positive. FN, false negative. TN, true negative. NR, not reported in the study

${ }^{a}$ The values represent the means \pm SD or medians with corresponding interquartile ranges (IQRs)

same criteria. For all these participants, pleural fluid samples were used in the Xpert MTB/RIF assay. The pooled sensitivity of Xpert MTB/RIF was 30\% (95\% CI: $21-42 \%, \mathrm{I}^{2}=87.93 \%$ ), while the pooled specificity was 99\% (95\% CI: $97-100 \%, I^{2}=96.20 \%$, Fig. 2). The SROC curve for Xpert MTB/RIF was situated near the desirable upper left corner of the plot (Fig. 3), and the area under the SROC curve (AUC) was 0.86 (95\% CI: 0.83-0.89).

\section{Accordance rate of rifampicin-susceptible and rifampicin- resistant cases}

Two of the 23 included studies presented the performance of Xpert MTB/RIF for the detection of rifampicin susceptibility and resistance in TP patients $[9,31]$. Comparison of the results between Xpert MTB/RIF and DST revealed a pooled accordance rate of was $99 \%\left(95 \% \quad \mathrm{CI}, \quad 95-104 \%, \quad \mathrm{I}^{2}=8.7 \%\right) \quad$ in rifampicin-susceptible cases, and $94 \%$ (95\% CI, 86$102 \%$ ) in rifampicin-resistant cases (Fig. 4). In one of these two studies [31], there was no rifampicin resistant case for either Xpert MTB/RIF or DST (Table 2), and thus the accordance rate was not calculated (Fig. 4b). These results indicate a trend of high concordance of Xpert MTB/RIF with DST for detection of rifampicin susceptibility and resistance, although the limited number of inclusive studies.

Table 2 Characteristics and results of Xpert MTB/RIF studies on rifampicin resistance

\begin{tabular}{|c|c|c|c|c|c|c|c|}
\hline \multirow{2}{*}{$\begin{array}{l}\text { First } \\
\text { author }\end{array}$} & \multirow[t]{2}{*}{ Year } & \multirow[t]{2}{*}{ Country } & \multirow{2}{*}{$\begin{array}{l}\text { Samples } \\
\text { enrolled }\end{array}$} & \multicolumn{2}{|l|}{ Xpert MTB/RIF } & \multicolumn{2}{|l|}{ DST } \\
\hline & & & & Rifampicin sensitive & Rifampicin resistant & Rifampicin sensitive & Rifampicin resistant \\
\hline Liu & 2015 & China & 43 & 32 & 11 & 33 & 10 \\
\hline Wang & 2015 & China & 13 & 13 & 0 & 13 & 0 \\
\hline
\end{tabular}




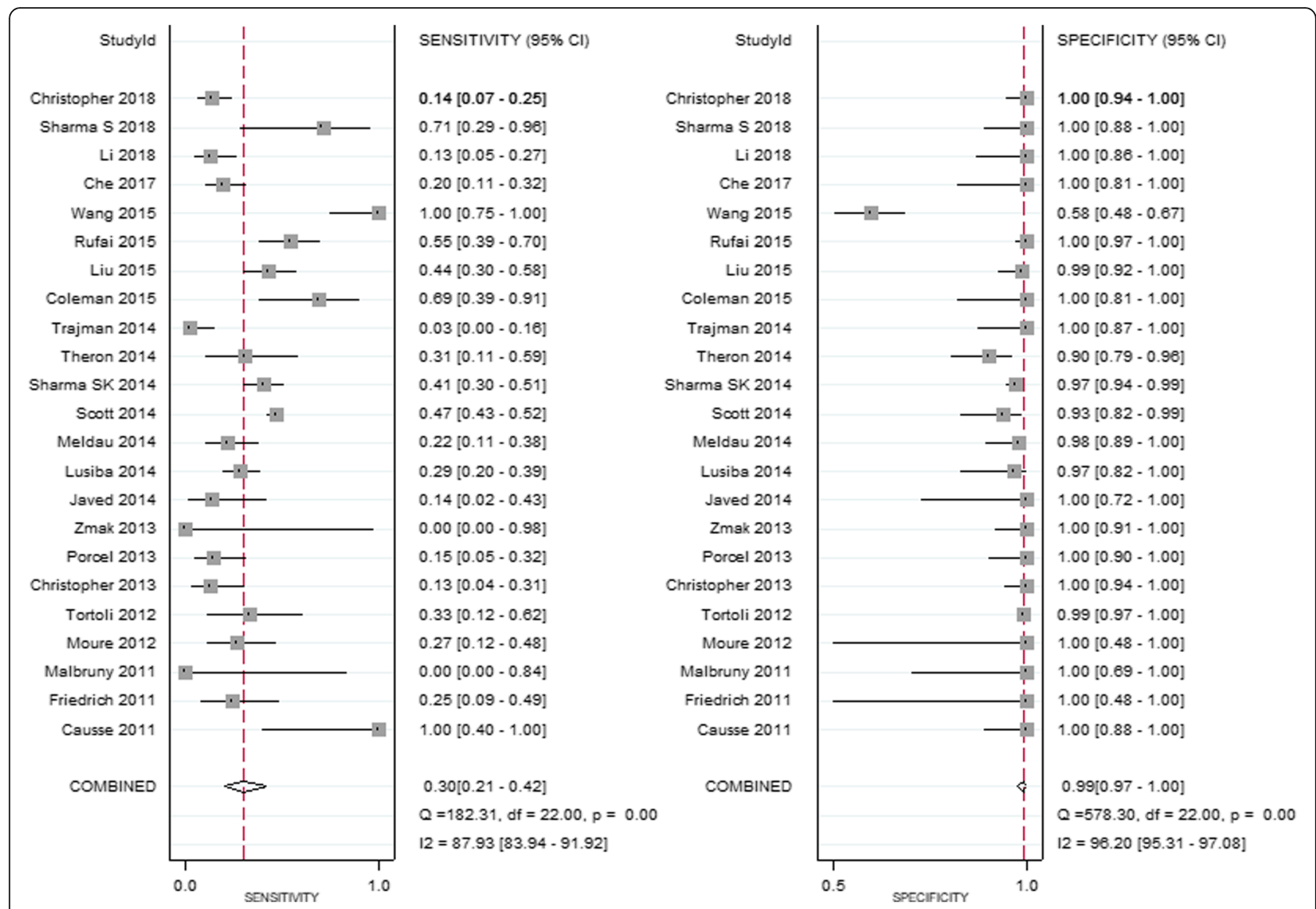

Fig. 2 Forest plots of the performance of Xpert MTB/RIF in diagnosing TP. See references $[5-9,19-36]$ for details

\section{Publication bias}

Based on Deeks' funnel plot asymmetry test, there was no significant asymmetry for the 23 studies on Xpert MTB/RIF $(P=0.54)$ included in our meta-analysis, suggesting a low risk for publication bias (Fig. 5).

\section{Discussion}

Despite an increasing and widespread use of Xpert MTB/RIF for the diagnosis of pulmonary and extra-pulmonary TB worldwide, it remains unclear how valuable this method is for diagnosing TB and detecting rifampicin resistance in TP patients using pleural fluid samples. To summarize the results of and to overcome the limitations of small samples size among existing individual studies, we conducted this systemic review and meta-analysis to assess the performance of Xpert MTB/ RIF in diagnosing TP and detecting rifampicin susceptibility or resistance using pleural fluid specimens. In order to produce the best possible results, we adopted a combination of histopathological examination and mycobacterial culture as the reference standard, which is the current gold standard for the diagnosis of TP. Our meta-analysis involves a total of 2646 patients with pleural effusion from 23 eligible studies published between 2011 and 2018 among 11 countries across Europe, South America, Asia and Africa (Table 1).

Based on our meta-analysis, the overall sensitivity and specificity of Xpert MTB/RIF in diagnosing TP from 23 eligible studies were 30 and $99 \%$, respectively, indicating a low sensitivity and a high specificity. While the high specificity of this assay suggests it to be an excellent rule-in test (confirming TP diagnosis), the low sensitivity suggests a limited rule-out value (ruling out TP diagnosis). The ability of Xpert MTB/RIF to detect rifampicin susceptibility or resistance was evaluated in two small studies, which showed a trend of high concordance with DST, suggesting its potential usefulness for detection of MDR-TB in TP patients.

A similar meta-analysis has been previously reported by Sehgal et al. [10], which reported a pooled sensitivity and specificity of 51.4 and $98.6 \%$, respectively, with culture used as a reference standard, and 22.7 and 99.8\%, respectively, with a composite reference standard (CRS) used as the benchmark. The specificity in this report is similar to that in our analysis (99\%) while the sensitivity in this report appears to be different compared to our 


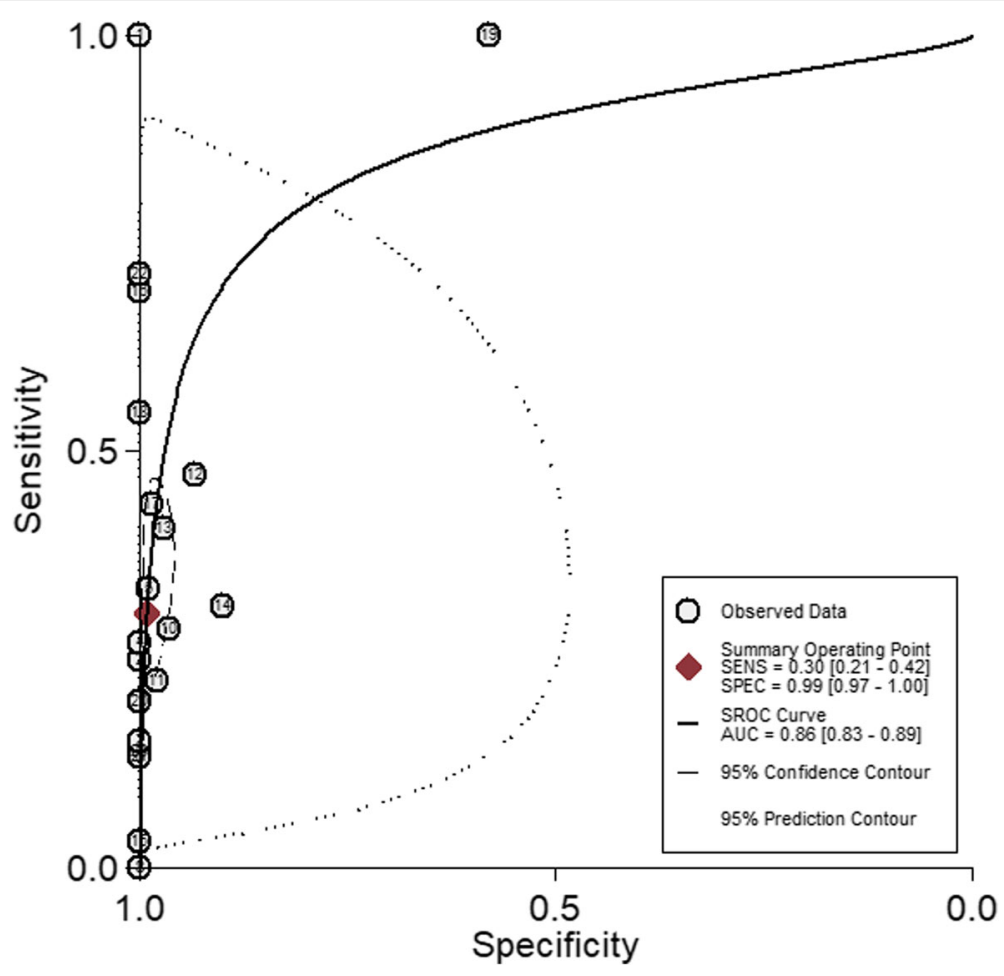

Fig. 3 Symmetric receiver operator characteristic (SROC) curve for Xpert MTB/RIF assay. The SROC curve was derived by Stata/MP 13.1

analysis (30\%). The exact reasons for the difference in sensitivity are unclear. One possible explanation is the lack of histopathological examination in the reference standard in the report of Sehgal et al. [10], which may lead to different results in sensitivity compared to our analysis. We used a combination of mycobacterial culture and histopathological examination as the reference standard, and excluded 9 studies included in the report of Sehgal et al. [10], which did not meet our reference standard while adding 8 new studies $[9,23$, $30,31,33-36]$ in our analysis. Given that the reference standard we used is the current gold standard, it is likely that the results of our analysis more reliable.

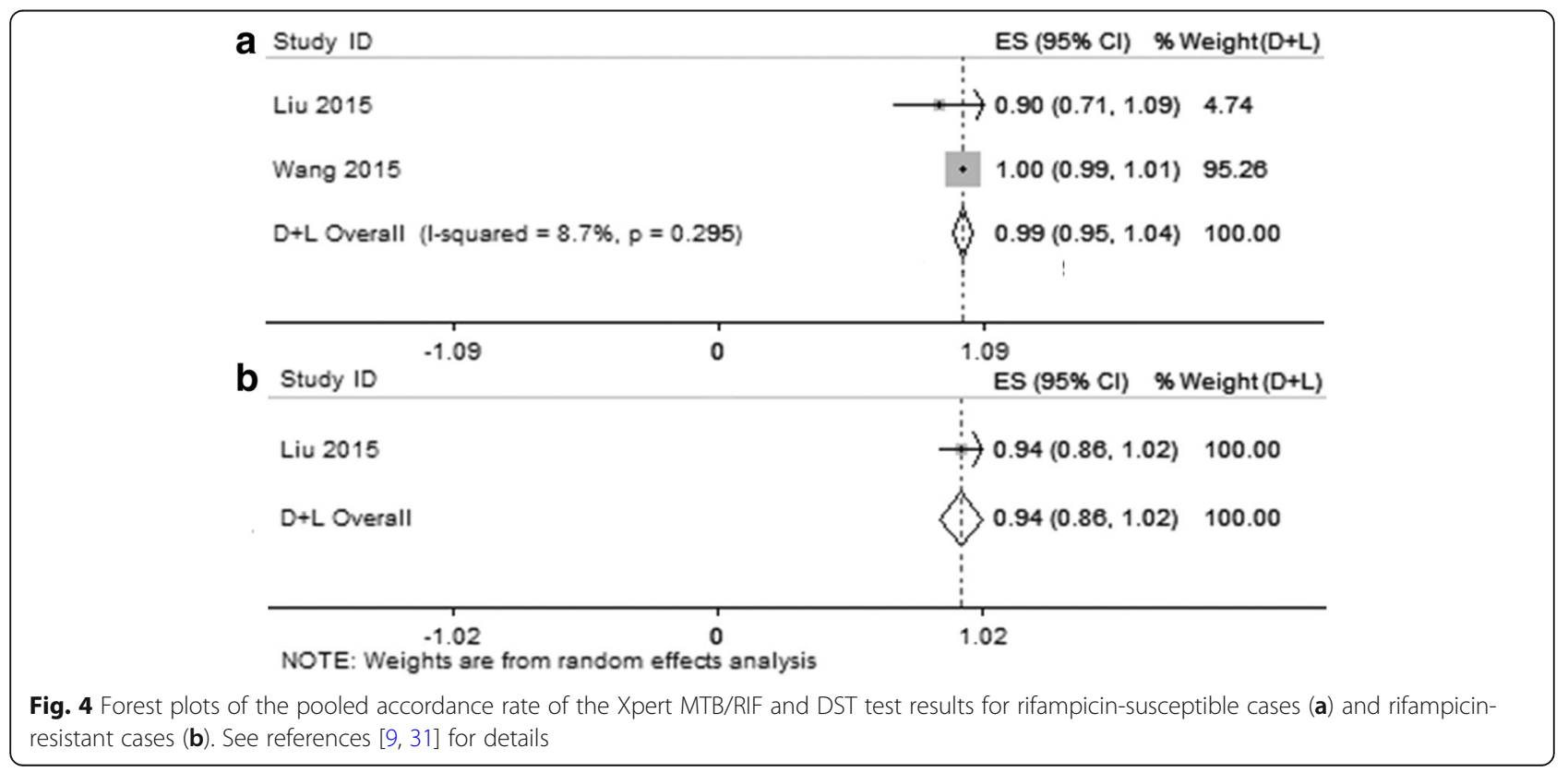




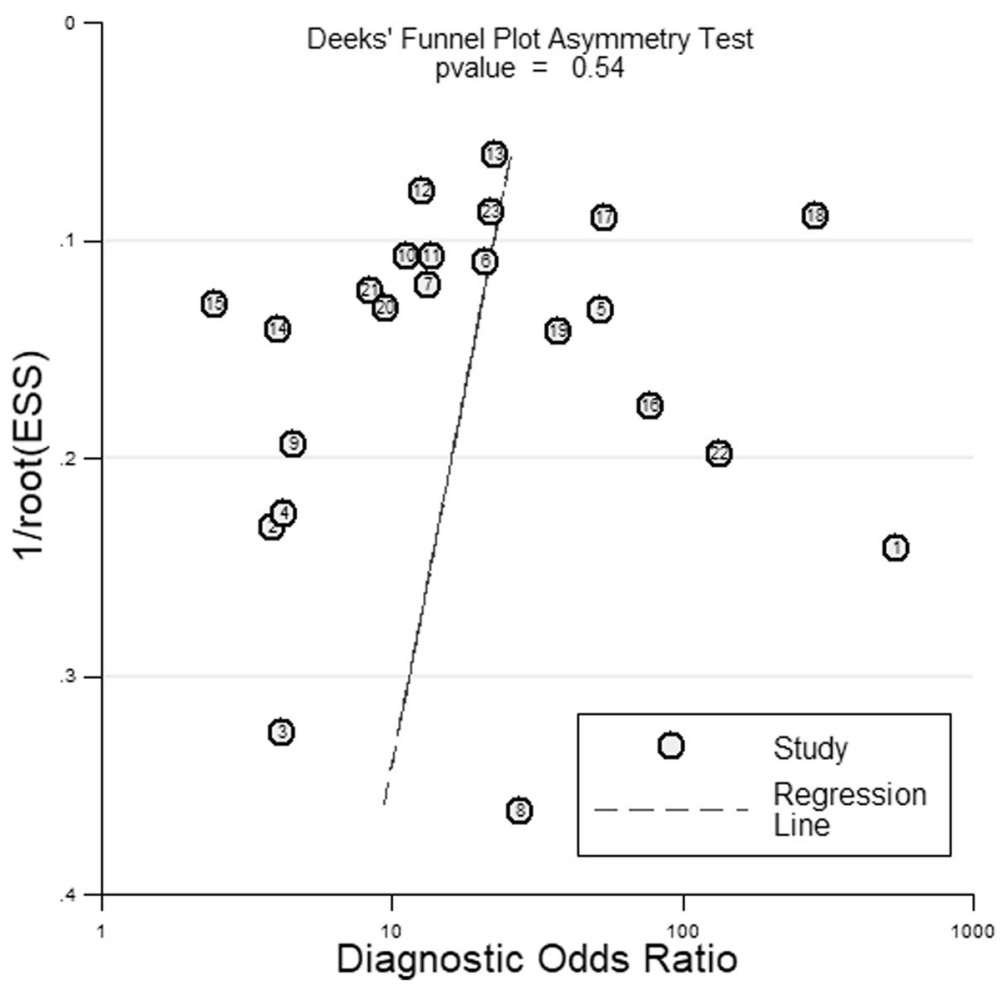

Fig. 5 A Deeks' funnel plot asymmetry test for evaluation of potential publication bias in Xpert MTB/RIF studies. This plot indicated a low risk of publication bias

Nevertheless, among the 23 studies included in our analysis there was substantial heterogeneity $\left(\mathrm{I}^{2}=87.93\right.$ and $96.20 \%$ for sensitivity and specificity, respectively). One prominent example is the study by Wang et al. [9], which showed an exceptionally high sensitivity but a very low specificity as clearly shown in the forest plots in Fig. 2. The reason for this observation is uncertain but could be related to the use of mycobacterial culture alone as the reference standard in this study. Since the mycobacterial culture method is known to have low sensitivity in TB diagnosis, evaluation of Xpert MTB/RIF using samples from patients confirmed by culture alone is likely to overestimate the sensitivity and underestimate the specificity, as has been reported previously $[5,9]$.

Although there is apparent variation in the sensitivity of Xpert MTB/RIF among different studies on TP patients, the overwhelming trend is a low sensitivity (around 30\%). When this assay is used as a diagnostic test, approximately $70 \%$ of TP patients could be misdiagnosed, suggesting that this assay is not appropriate as an initial screening test for suspected TP patients in countries with high TB burden. The reasons for this low sensitivity remain poorly understood, but could be due to the presence of PCR inhibitors in pleural fluid samples, and use of inappropriate or inefficient sampling methods [37]. Clearly, further research is needed to optimize the sample processing in order to improve the sensitivity of Xpert MTB/RIF.

Despite a low sensitivity for diagnosing TP, Xpert MTB/RIF consistently showed an excellent specificity (99\%), which is attributed largely to the use of highly specific genetic target in this test. The high specificity suggests its high value in confirming TP diagnosis and differentiating TP from non-TB diseases, especially in countries with low or intermediate TB burden.

For the Xpert MTB/RIF assay in detection of rifampicin resistance in pleural fluid samples, the pooled accordance rate of rifampicin-susceptible cases and rifampicin-resistant cases was 99 and 94\%, separately. The high concordance between the Xpert MTB/RIF and DST indicated good efficiency for rifampicin resistance detection, which was similar to the previous studies [38-40]. Although the number of inclusive studies in our meta-analysis is limited, the result indicated the Xpert MTB/RIF could rapidly detect patients suffered from MDR-TB and give them rapid initiation of anti-MDR-TB therapy.

The Deeks' funnel plot asymmetry test was performed to evaluate and analyze the publication bias among 23 inclusive studies (Fig. 5). The plot showed no significant asymmetry for the Xpert MTB/RIF $(P=0.54)$, and there was no evidence of potential risk of publication bias. 


\section{Conclusions}

In summary, the results of our meta-analysis suggest that the Xpert MTB/RIF assay is of limited value as a screening test for TP but has a high potential for confirming TP diagnosis and differentiating TP from non-TB diseases. The Xpert MTB/RIF assay showed high concordance with DST, suggesting its usefulness for detection of MDR-TB, which may help early decision making in anti-MDR-TB therapy.

\section{Additional file}

Additional file 1: Methodological quality evaluation results of 23 studies sorted using the Quality Assessment of Diagnostic Accuracy Studies-2 (QUADAS-2) tool. The ratings of risk of bias and applicability concerns were provided. (TIF $3222 \mathrm{~kb}$ )

\section{Abbreviations}

95\% Cl: 95\% confidence intervals; AUC: Area under the SROC curve DST: Drug susceptibility testing; MDR-TB: Multiple drug resistant-TB; PCR: Polymerase chain reaction; PRISMA: Preferred reporting items for systematic reviews and meta-analyses; QUADAS-2: Quality Assessment of Diagnostic Accuracy Studies-2; RIF: Rifampin; SROC: Symmetric receiver operator characteristic; TB: Tuberculosis; TP: Tuberculous pleurisy; WHO: World Health Organization

\section{Acknowledgements}

We sincerely thank all authors who provided published data for our metaanalysis, and we also acknowledge the editors and reviewers for insightful suggestions on this work. We thank Prof. Bin Peng and Dr. Youlun Li for thei help in statistics and diagnostic criteria.

\section{Funding}

This work was supported by the National Key Clinical Specialist Construction Programs of China [2012] NO. 649. The funders had no role in study design, data collection and analysis, decision to publish, or preparation of the manuscript.

\section{Availability of data and materials}

We declare that the data supporting the conclusions of this article are fully described within the article.

\section{Authors' contributions}

$\mathrm{ZH}$ and LP conceived and designed the experiments. $\mathrm{ZH}$ and LP analyzed the data. ZH and LP performed the experiments. ZH and LP wrote the paper Both authors contributed equally to preparing the manuscript and approve of the content.

\section{Ethics approval and consent to participate}

All analyses were based on previous published studies, thus no ethical approval and patient consent are required.

\section{Consent for publication}

Not applicable.

\section{Competing interests}

All authors declare no competing interests. No competing interests exists in this study due to commercial or other associations (e.g., pharmaceutical stock ownership, consultancy, advisory board membership, relevant patents, or research funding). No competing interests exists in the submission of this manuscript, and the manuscript is approved by all of the authors for publication, all authors that the work described was original research that has not been published previously and is not under consideration for publication elsewhere in whole or in part.

\section{Publisher's Note}

Springer Nature remains neutral with regard to jurisdictional claims in published maps and institutional affiliations.

Received: 28 November 2016 Accepted: 18 June 2018

Published online: 25 June 2018

\section{References}

1. World Health Organization. Global tuberculosis report 2017. Available: http://apps.who.int/iris/bitstream/10665/259366/1/9789241565516-eng.pdf. Accessed 26 May 2018.

2. Light RW. Update on tuberculous pleural effusion. Respirology. 2010;15:4518. https://doi.org/10.1111/j.1440-1843.2010.01723.x.

3. World Health Organization. Automated real-time nucleic acid amplification technology for rapid and simultaneous detection of tuberculosis and rifampicin resitance: Xpert MTB/RIF System. Available: http://whqlibdoc.who. int/publications/2011/9789241501545_eng.pdf. Accessed 26 May 2018.

4. World Health Organization. Automated real-time nucleic acid amplification technology for rapid and simultaneous detection of tuberculosis and rifampicin resistance: Xpert MTB/RIF system for the diagnosis of pulmonary and extrapulmonaryTB in adults and children. Available: http://apps.who.int/ iris/bitstream/10665/112472/1/9789241506335_eng.pdf. Accessed 26 May 2018.

5. Causse M, Ruiz P, Gutiérrez-Aroca JB, Casal M. Comparison of two molecular methods for rapid diagnosis of extrapulmonary tuberculosis. J Clin Microbiol. 2011:49:3065-7. https://doi.org/10.1128/JCM.00491-11.

6. Malbruny B, Le Marrec G, Courageux K, Leclerca R, Cattoir V. Rapid and efficient detection of Mycobacterium tuberculosis in respiratory and nonrespiratory samples. Int J Tuberc Lung Dis. 2011;15:553-5. https://doi.org/10. 5588/ijtld.10.0497.

7. Tortoli E, Russo C, Piersimoni C, Mazzola E, Dal Monte P, Pascarella M, et al. Clinical validation of Xpert MTB/RIF for the diagnosis of extrapulmonary tuberculosis. Eur Respir J. 2012;40:442-7. https://doi.org/10.1183/09031936. 00176311

8. Christopher DJ, Schumacher SG, Michael JS, Luo R, Balamugesh T, Duraikannan P. Performance of Xpert MTB/RIF on pleural tissue for the diagnosis of pleural tuberculosis. Eur Respir J. 2013:42:1427-9. https://doi. org/10.1183/09031936.00103213.

9. Wang D, Chen Z, Chen Q, Zhang G. Evaluation of GeneXpert MTB/RIF assay for diagnosis of tuberculous pleurisy and detection of rifampin resistance. Int J Respir. 2015;35:1071-3. https://doi.org/10.3760/cma.j.issn.1673-436X. 2015.14.007

10. Sehgal IS, Dhooria S, Aggarwal AN, Behera D, Agarwal R. Diagnostic performance of Xpert MTB/RIF in tuberculous pleural effusion: systematic review and meta-analysis. J Clin Microbiol. 2016;54:1133-6. https://doi.org/ 10.1128/JCM.03205-15

11. Moher D, Liberati A, Tetzlaff J, Altman DG, PRISMA Group. Preferred reporting items for systematic reviews and meta-analyses: the PRISMA statement. PLoS Med. 2009;6:e1000097. https://doi.org/10.1371/journal. pmed.1000097.

12. Udwadia ZF, Sen T. Pleural tuberculosis: an update. Curr Opin Pulm Med. 2010;16:399-406. https://doi.org/10.1097/MCP.0b013e328339cf6e.

13. Whiting PF, Rutjes AW, Westwood ME, Mallett S, Deeks JJ, Reitsma JB, et al. QUADAS-2: a revised tool for the quality assessment of diagnostic accuracy studies. Ann Intern Med. 2011;155:529-36. https://doi.org/10.7326/00034819-155-8-201110180-00009.

14. Devillé WL, Buntinx F, Bouter LM, Montori VM, de Vet HC, van der Windt DA, et al. Conducting systematic reviews of diagnostic studies: didactic guidelines. BMC Med Res Methodol. 2002;2:9. https://doi.org/10.1186/14712288-2-9.

15. Leeflang MM, Deeks JJ, Gatsonis C, Bossuyt PM. Cochrane diagnostic test accuracy working group. Systematic reviews of diagnostic test accuracy. Ann Intern Med. 2008;149:889-97. https://doi.org/10.7326/0003-4819-14912-200812160-00008.

16. Moses LE, Shapiro D, Littenberg B. Combining independent studies of a diagnostic test into a summary ROC curve: data-analytic approaches and some additional considerations. Stat Med 1993; 12: 1293-1316. PMID: 8210827.

17. Irwig L, Macaskill P, Glasziou P, Fahey M. Meta-analytic methods for diagnostic test accuracy. J Clin Epidemiol. 1995;48:119-30. https://doi.org/ 10.1016/0895-4356(94)00099-C. 
18. Vamvakas EC. Meta-analyses of studies of the diagnostic accuracy of laboratory tests: a review of the concepts and methods. Arch Pathol Lab Med. 1998;122:675-86. 9701328

19. Friedrich SO, Von Groote-Bidlingmaier F, Diacon AH. Xpert MTB/RIF assay for diagnosis of pleural tuberculosis. J Clin Microbiol. 2011;49:4341-2. https:// doi.org/10.1128/JCM.05454-11.

20. Moure R, Martín R, Alcaide F. Effectiveness of an integrated real-time PCR method for detection of the Mycobacterium tuberculosis complex in smearnegative extrapulmonary samples in an area of low tuberculosis prevalence. J Clin Microbiol. 2012;50:513-5. https://doi.org/10.1128/JCM.06467-11.

21. Porcel JM, Palma R, Valdés L, Bielsa S, San-José E, Esquerda A. Xpert ${ }^{\circledast}$ MTB/ RIF in pleural fluid for the diagnosis of tuberculosis. Int J Tuberc Lung Dis. 2013;17:1217-9. https://doi.org/10.5588/ijtld.13.0178.

22. Zmak L, Jankovic M, Jankovic VK. Evaluation of Xpert MTB/RIF assay for rapid molecular diagnosis of tuberculosis in a two-year period in Croatia. Int J Mycobacteriol. 2013;2:179-82. https://doi.org/10.1016/j.jimyco.2013.05.003.

23. Javed N, Aslam M, Mushtaq MA, Khan T, Shaheen MZ. Role of gene Xpert in diagnosis of tuberculous pleural effusion: comparison with pleural biopsy. Eur Respir J. 2014;44(Suppl 58):P2655.

24. Lusiba JK, Nakiyingi L, Kirenga BJ, Kiragga A, Lukande R, Nsereko M, et al. Evaluation of Cepheid's Xpert MTB/RIF test on pleural fluid in the diagnosis of pleural tuberculosis in a high prevalence HIV/TB setting. PLoS One. 2014; 9:e102702. https://doi.org/10.1371/journal.pone.0102702.

25. Meldau R, Peter J, Theron G, Calligaro G, Allwood B, Symons G, et al. Comparison of same day diagnostic tools including gene Xpert and unstimulated IFN- $\gamma$ for the evaluation of pleural tuberculosis: a prospective cohort study. BMC Pulm Med. 2014;14:58. https://doi.org/10.1186/1471-2466-14-58.

26. Scott LE, Beylis N, Nicol M, Nkuna G, Molapo S, Berrie L, et al. Diagnostic accuracy of xpert MTB/RIF for extrapulmonary tuberculosis specimens: establishing a laboratory testing algorithm for South Africa. J Clin Microbiol. 2014;52:1818-23. https://doi.org/10.1128/JCM.03553-13.

27. Sharma SK, Kohli M, Chaubey J, Yadav RN, Sharma R, Singh BK, et al. Evaluation of Xpert MTB/RIF assay performance in diagnosing extrapulmonary tuberculosis among adults in a tertiary care Centre in India. Eur Respir J. 2014;44:1090-3. https://doi.org/10.1183/09031936.00059014.

28. Theron G, Peter J, Calligaro G, Meldau R, Hanrahan C, Khalfey H, et al. Determinants of PCR performance (Xpert MTB/RIF), including bacterial load and inhibition, for TB diagnosis using specimens from different body compartments. Sci Rep. 2014;4:5658. https://doi.org/10.1038/srep05658.

29. Trajman A, De Oliveira EF DSSK, Bastos ML, Belo Neto E, Silva EM, Da Silva Lourenço MC, et al. Accuracy of polimerase chain reaction for the diagnosis of pleural tuberculosis. Respir Med. 2014;108:918-23. https://doi.org/10. 1016/j.rmed.2014.04.007.

30. Coleman M, Finney L, Komrower D, Chitani A, Bates J, Chipungu GA, et al. Markers to differentiate between Kaposi's sarcoma and tuberculous pleural effusions in HIV-positive patients. Int J Tuberc Lung Dis. 2015;19:144-50. https://doi.org/10.5588/ijtld.14.0289.

31. Liu X, Huang Z, Du J. Rapid diagnosis of pleural tuberculosis by Xpert MTB/ RIF assay. Zhonghua jie he he hu xi za zhi. 2015:38:741-5. https://doi.org/10. 3760/cma.j.issn.1001-0939.2015.10.005.

32. Rufai $S B$, Singh A, Kumar $P$, Singh J, Singh S. Performance of xpert MTB/RIF assay in diagnosis of pleural tuberculosis by use of pleural fluid samples. J Clin Microbiol. 2015:53:3636-8. https://doi.org/10.1128/JCM.02182-15.

33. Che N, Yang X, Liu Z, Li K, Chen X. Rapid detection of cell-free Mycobacterium tuberculosis DNA in tuberculous pleural effusion. J Clin Microbiol. 2017;55:1526-32. https://doi.org/10.1128/JCM.02473-16.

34. Li XL, Chen HM, Zhang LQ, Ma LP, WU XG, MQ GAO. Diagnostic value of three kinds of pleural effusion detection methods for pleural tuberculous. Chinese J Med. 2018;53:134-8. https://doi.org/10.3969/j.issn.1008-1070.2018. 02.005 .

35. Sharma S, Dahiya B, Sreenivas V, Singh N, Raj A, Sheoran A, et al. Comparative evaluation of GeneXpert MTB/RIF and multiplex PCR targeting mpb64 and IS6110 for the diagnosis of pleural TB. Future Microbiol. 2018;13: 407-13. https://doi.org/10.2217/fmb-2017-0147.

36. Christopher DJ, Dinakaran S, Gupta R, James P, Isaac B, Thangakunam B. Thoracoscopic pleural biopsy improves yield of Xpert MTB/RIF for diagnosis of pleural tuberculosis. Respirology. 2018; https://doi.org/10.1111/resp.13275.

37. Weyer K, Mirzayev F, Migliori GB, Van Gemert W, D'Ambrosio L, Zignol M, et al. Rapid molecular TB diagnosis: evidence, policy-making and global implementation of Xpert MTB/RIF. Eur Respir J. 2013:42:252-71. https://doi. org/10.1183/09031936.00157212.
38. Chang K, Lu W, Wang J, Zhang K, Jia S, Li F, et al. Rapid and effective diagnosis of tuberculosis and rifampicin resistance with Xpert MTB/RIF assay: a meta-analysis. J Inf Secur. 2012;64:580-8. https://doi.org/10.1016/j. jinf.2012.02.012

39. Steingart KR, Schiller I, Horne DJ, Pai M, Boehme CC, Dendukuri N. Xpert ${ }^{\circledast}$ MTB/RIF assay for pulmonary tuberculosis and rifampicin resistance in adults. Cochrane Database Syst Rev. 2014;21:CD009593. https://doi.org/10. 1002/14651858.CD009593.pub3.

40. Wang XW, Pappoe F, Huang Y, Cheng XW, Xu DF, Wang H, et al. Xpert MTB/RIF assay for pulmonary tuberculosis and rifampicin resistance in children: a meta-analysis. Clin Lab. 2015;61:1775-85. 26732005

\section{Ready to submit your research? Choose BMC and benefit from:}

- fast, convenient online submission

- thorough peer review by experienced researchers in your field

- rapid publication on acceptance

- support for research data, including large and complex data types

- gold Open Access which fosters wider collaboration and increased citations

- maximum visibility for your research: over $100 \mathrm{M}$ website views per year

At BMC, research is always in progress.

Learn more biomedcentral.com/submissions 